OPEN ACCESS

Edited by:

Gaetano Santulli,

Columbia University, USA

Reviewed by:

Vincent Jacquemet,

Université de Montréal, Canada

Wenjun Xie,

Xi'an Jiaotong University, China

*Correspondence:

Oleg V. Aslanidi

oleg.aslanidi@kcl.ac.uk

Specialty section: This article was submitted to

Cardiac Electrophysiology,

a section of the journal

Frontiers in Physiology

Received: 13 August 2016 Accepted: 03 October 2016 Published: 25 October 2016

Citation:

Morgan R, Colman MA, Chubb H, Seemann $G$ and Aslanidi OV (2016)

Slow Conduction in the Border Zones of Patchy Fibrosis Stabilizes the Drivers for Atrial Fibrillation: Insights from Multi-Scale Human Atrial Modeling. Front. Physiol. 7:474 doi: 10.3389/fphys.2016.00474

\section{Slow Conduction in the Border Zones of Patchy Fibrosis Stabilizes the Drivers for Atrial Fibrillation: Insights from Multi-Scale Human Atrial Modeling}

\author{
Ross Morgan ${ }^{1}$, Michael A. Colman ${ }^{2}$, Henry Chubb ${ }^{1}$, Gunnar Seemann ${ }^{3}$ and \\ Oleg V. Aslanidi ${ }^{1 *}$
}

${ }^{1}$ Division of Imaging Sciences and Biomedical Engineering, Department of Biomedical Engineering, King's College London, London, UK, ${ }^{2}$ School of Biomedical Sciences, University of Leeds, Leeds, UK, ${ }^{3}$ Institute for Experimental Cardiovascular Medicine, University Heart Center - Bad Krozingen, Medical Center - University of Freiburg, Freiburg, Germany

Introduction: The genesis of atrial fibrillation (AF) and success of AF ablation therapy have been strongly linked with atrial fibrosis. Increasing evidence suggests that patient-specific distributions of fibrosis may determine the locations of electrical drivers (rotors) sustaining AF, but the underlying mechanisms are incompletely understood. This study aims to elucidate a missing mechanistic link between patient-specific fibrosis distributions and AF drivers.

Methods: 3D atrial models integrated human atrial geometry, rule-based fiber orientation, region-specific electrophysiology, and AF-induced ionic remodeling. A novel detailed model for an atrial fibroblast was developed, and effects of myocyte-fibroblast (M-F) coupling were explored at single-cell, 1D tissue and 3D atria levels. Left atrial LGE $\mathrm{MRI}$ datasets from 3 chronic AF patients were segmented to provide the patient-specific distributions of fibrosis. The data was non-linearly registered and mapped to the 3D atria model. Six distinctive fibrosis levels (0-healthy tissue, 5-dense fibrosis) were identified based on LGE MRI intensity and modeled as progressively increasing M-F coupling and decreasing atrial tissue coupling. Uniform 3D atrial model with diffuse (level 2) fibrosis was considered for comparison.

Results: In single cells and tissue, the largest effect of atrial M-F coupling was on the myocyte resting membrane potential, leading to partial inactivation of sodium current and reduction of conduction velocity (CV). In the 3D atria, further to the M-F coupling, effects of fibrosis on tissue coupling greatly reduce atrial CV. AF was initiated by fast pacing in each 3D model with either uniform or patient-specific fibrosis. High variation in fibrosis distributions between the models resulted in varying complexity of $A F$, with several drivers emerging. In the diffuse fibrosis models, waves randomly meandered through the atria, whereas in each the patient-specific models, rotors stabilized in fibrotic regions. The rotors propagated slowly around the border zones of patchy fibrosis (levels 3-4), failing to spread into inner areas of dense fibrosis. 
Conclusion: Rotors stabilize in the border zones of patchy fibrosis in 3D atria, where slow conduction enable the development of circuits within relatively small regions. Our results can provide a mechanistic explanation for the clinical efficacy of ablation around fibrotic regions.

Keywords: atrial fibrillation, fibrosis, modeling and simulation, arrhythmia mechanisms, MR imaging

\section{INTRODUCTION}

Atrial fibrillation (AF) is the most common sustained cardiac arrhythmia, affecting 6 million people in Europe alone and imposing a huge healthcare burden on the society. Despite well-developed clinical guidelines for the management of AF, anti-arrhythmic therapy success rates remain suboptimal due to complexity of the disease and lack of knowledge of its mechanisms (Calkins et al., 2012). Catheter ablation is a standard clinical treatment aimed at eliminating the arrhythmogenic electrical drivers in the atria by destroying the underlying tissue substrate with a localized energy delivery. However, precise driver locations in a patient are generally unknown, and the longterm success rates of empirical ablation procedures (such as the pulmonary vein isolation) are low, with the disease recurring in $30-50 \%$ of patients (Schotten et al., 2011; Calkins et al., 2012).

Both the genesis of AF and success of ablation therapy have been strongly linked with atrial fibrosis (Marrouche et al., 2014; Gal and Marrouche, 2015; Kottkamp et al., 2015). Fibrosis levels are higher in AF patients compared to healthy subjects and correlate positively with AF recurrence (Marrouche et al., 2014) after ablation. Moreover, ablation around fibrotic areas identified from late-gadolinium enhanced (LGE) MRI (Gal and Marrouche, 2015) or electro-anatomical mapping (Kottkamp et al., 2015) has been shown to improve therapy success rates. Mechanistically, structural and functional tissue heterogeneities associated with fibrosis have generally been linked to arrhythmogenesis, for example in ventricular infarction border zones (BZ) (Rutherford et al., 2012). Such border heterogeneities can be presented as large collagen deposits within the functional myocardium, which can slow down or block the propagation of electrical excitation waves, creating conditions for the generation and sustenance of re-entrant drivers (rotors).

Recent studies (Jadidi et al., 2013; Haissaguerre et al., 2016) have demonstrated that areas adjacent to dense fibrosis in the atria have also shown high levels of arrhythmogenic activity. These myocardial areas, whilst still fibrotic, are not damaged sufficiently to fully inhibit wave propagation and have a high correlation to complex high-frequency electrical activity (Jadidi et al., 2013). Structurally and functionally these regions are reminiscent of ventricular infarction BZs, which commonly surround dense fibrotic areas (such as scar) and have also been shown to exhibit low levels of electrical activity. This similarity suggests that patient-specific distributions of atrial fibrosis can also determine the dynamics of electrical drivers sustaining AF. A recent computational study has shown that $\mathrm{AF}$ can be perpetuated by rotors persisting in fibrotic BZs with specific statistical characteristics (Zahid et al., 2016). However, mechanistic links between atrial fibrosis and the genesis of AF drivers are unclear.

Computational modeling provides a powerful tool for integrating multi-scale atrial data and dissecting the mechanisms of arrhythmia and anti-arrhythmic treatments in-silico, (Colman et al., 2013; McDowell et al., 2015; Zahid et al., 2016) which is extremely challenging in a purely experimental or clinical setting. Recent computational studies have shown the importance of heterogeneities associated with fibrosis, (McDowell et al., 2015; Zahid et al., 2016) as well as other factors, (Aslanidi et al., 2011; Colman et al., 2013) in the generation and sustenance of AF. However, even the most advanced models have only considered step-wise heterogeneities, such as discontinuous transitions from healthy or fibrotic tissue with no genuine transitional zone (Zahid et al., 2016)-and hence, have not explored the mechanistic effects of patchy fibrotic BZs. Besides, electrophysiological models developed for ventricular (rather than atrial) fibroblasts have commonly been utilized (McDowell et al., 2015).

The current study aims to develop both a novel model for atrial fibroblast, and a novel image-based approach to modeling gradual transitions in properties between healthy and fibrotic atrial tissue. These novel electrophysiological and structural data will be integrated into multi-scale biophysical models of the 3D human atria. The detailed models will then be applied to substantiate mechanistic links between patient-specific distributions of fibrosis and location of drivers sustaining AF. Specifically, we will explore a hypothesis that rotors preferentially propagate around the slow conducting BZs of patchy fibrosis, which can provide a mechanistic explanation for the improved outcomes of ablation around fibrotic areas.

\section{METHODS}

Several authors of this study have recently contributed to the creation of an integrative biophysical model for the entire 3D human atria (Aslanidi et al., 2011; Colman et al., 2013). The model included: (i) detailed atrial electrophysiology based on the Courtemanche-Ramirez-Nattel (Courtemanche et al., 1998) (CRN) atrial myocyte model; (ii) regional heterogeneity in the ionic channel and action potential (AP) properties, and their changes due to AF-induced ionic remodeling, reflected in the respective variations of the CRN model parameters; (iii) atrial geometry provided by the Visible Female anatomical dataset, with the $3 \mathrm{D}$ atria segmented into major anatomical regions (Colman et al., 2013). In the current study, the model was further developed to include: (iv) rule-based fiber orientation throughout the entire $3 \mathrm{D}$ atria (see in the Supplementary Figure 1; Krueger et al., 2011); (v) a novel model for atrial 
fibroblast electrophysiology and electrotonic myocyte-fibroblast (M-F) coupling; (vi) patient-specific distributions of fibrosis reconstructed from LGE MRI data and mapped onto the 3D atria model, which accounted for gradual changes of the tissue properties between healthy and fibrotic atrial regions. Detailed description of the existing model components (i-iii), as well as (iv), can be found in previous publications (Aslanidi et al., 2011; Krueger et al., 2011; Colman et al., 2013), and the novel components (v,vi) are described in detail below.

\section{Atrial Fibroblast Electrophysiology}

Unlike myocytes, fibroblasts are not electrically excitable, however they can maintain a resting membrane potential (RMP), and hence affect and be affected by the tissue electrophysiology. The electrophysiological function of fibroblasts is determined by the presence of a range of ionic channel currents in their cell membrane. It is known that both cultured and freshly isolated fibroblasts express voltage dependant potassium channels $\left(I_{K v}\right)$ (Chilton et al., 2005). A wide variety of currents have now been discovered in the cardiac fibroblast, predominantly potassium $\left(I_{K u r}, I_{K i r}, I_{t o}\right)$, but also non-specific ionic currents $\left(I_{n s}\right)$ which can be mechanically and non-mechanically activated chlorine currents $\left(I_{C l}\right)$. The sources of data for these various currents are diverse, and are summarized in Table 1.

The general approach to modeling atrial fibroblast electrophysiology was based on a well-established ventricular fibroblast model by MacCannell et al. (2007). However, a set of ionic channel currents specific to atrial fibroblasts was considered, based on the CRN formulation of the currents for human atrial myocytes and experimental data from atrial fibroblasts. Data for the atrial fibroblast model was obtained from a study (Wu et al., 2014) on ionic currents in freshly isolated canine atrial fibroblasts. An optimal fit between the modeled ionic currents and experimental data was achieved by varying the current conductances (see Results below). Note that the dog has been widely used as an experimental model for AF (Schotten et al., 2011), and in the absence of ionic current data from human fibroblasts it is sensible to propose that the canine data provides the closest match.

The 4-aminopyridine (4-AP) sensitive current was modeled as $I_{t o, f}$, which is selectively blocked by 4-AP. Fitting the currentvoltage (I-V) relationship for this current to experimental data by $\mathrm{Wu}$ et al. (2014) involved a 90\% reduction in the current conductance compared to its original formulation in the CRN model, but no changes to the CRN current kinetics was introduced.

The $S 9941$ sensitive current was modeled as $I_{K u r, f}$, as $S 9941$ is a blocker of ionic channels containing the subunit Kv1.5, which forms the channel conducting this current. Fitting the I$\mathrm{V}$ relationship for this current to experimental data by Wu et al. (2014) involved a $40 \%$ reduction in the current conductance compared to its original formulation in the CRN model.

The end pulse current was measured by Wu et al. (2014) as the total voltage sensitive current. We considered the remainder of this current after subtracting both $I_{t o, f}$ and $I_{K u r, f}$ as a sum of a further two currents: a linear non-specific ionic channel current, $I_{n s, f}$, and the inward-rectifier potassium channel current, $I_{K 1, f}$ (see Results below). Both $I_{K u r, f}$ and $I_{n s, f}$ are major contributors to the fibroblast RMP. The separation of these two currents ensured that $I_{K 1, f}$ reversed at the equilibrium Nernst potential for potassium ions, $E_{K}$, as is observed in electrophysiological recordings of this current in multiple cell types. The voltage and time dependences for these currents are based on the CRN formulation (index $f$ denotes their relevance to fibroblasts):

$$
\begin{aligned}
I_{K 1, f} & =\frac{0.03\left(V_{f}+86.75\right)}{\left(1+\exp \left(0.05\left(V_{f}+20\right)\right)\right)} \\
I_{n s, f} & =0.018 V_{f} \\
I_{t o, f} & =g_{t o, f} * o_{a, f^{3}} * o_{i, f} *\left(V_{f}-E_{K}\right) ; \\
I_{K u r, f} & =g_{K u r, f} * u_{a, f}{ }^{3} * u_{i, f} *\left(V_{f}-E_{K}\right) ;
\end{aligned}
$$

Here, $V_{f}$ is the membrane voltage of fibroblasts, where $o_{a, f}, u_{a, f}$ and $o_{i, f}, u_{i, f}$ are activation and inactivation gates, respectively, and $g_{K u r}$ and $g_{\text {to }}$ are conductances of the respective channels.

The other two currents in the developed atrial fibroblast model are based on the MacCannell et al. (2007) formulation. Specifically, there are currents not measured by Wu et al. (2014) but assumed present in the atrial fibroblast based on experimental studies with other cardiac cells. These are the sodium-potassium exchange current $\left(I_{\mathrm{NaK}}\right)$ and the background sodium current $\left(I_{b N a}\right)$.

Note that in the MacCannell et al. (2007) model and its derivatives, only $I_{K 1}$ and the time and voltage dependant potassium current $I_{K v}$ are included. The latter current was shown to be sensitive to 4-AP and S9941 in their experimental work, (Shibukawa et al., 2005) and hence is likely to present a sum of $I_{t o, f}$ and $I_{K u r, f}$. However, unlike our novel model, MacCannell et al. (2007) modeled it as a single current.

$\mathrm{M}-\mathrm{F}$ electrotonic coupling mechanism is based on the presence of M-F gap junctions (Rook et al., 1992; Gaudesius et al., 2003; Camelliti et al., 2004), in the same manner as inter-myocyte coupling. In order to simulate the M-F coupling, we used a similar formalism to that developed by MacCannell et al. (2007). Specifically, the CRN atrial myocyte (with the total membrane ionic current $I_{t o t}$ ) was coupled with the novel atrial fibroblast (with a total membrane ionic current $I_{t o t, f}$ ) via a linear M-F gap junctional conductance $\left(G_{g a p}\right)$ :

$$
\begin{array}{r}
I_{\text {tot }, f}=\sum I_{\text {ion }, f}=I_{t o, f}+I_{K 1, f}+I_{K u r, f}+I_{n s, f} \\
+I_{b N a, f}+I_{N a K, f} \\
\frac{d V_{f}}{d t}=-\frac{1}{C_{m f}}\left[I_{t o t, f}\left(V_{f}, t\right)+G_{g a p}\left(V_{f}-V_{m}\right)\right] \\
\frac{d V_{m}}{d t}=-\frac{1}{C_{m}}\left[I_{\text {tot }}\left(V_{m}, t\right)+\sum_{i=1}^{n} G_{g a p}\left(V_{m}-V_{f}\right)\right]
\end{array}
$$

Here $C_{m}$ is the membrane capacitance, subscripts $m$ and $f$ relate to a myocyte and fibroblast, and $n$ to the number of fibroblasts coupled to one myocyte. $G_{g a p}$ was set as $0.5 \mathrm{nS}$ (Jacquemet and Henriquez, 2008) and $n$ was varied between 0 and 6 . Unless specified otherwise, a myocyte was coupled to 2 fibroblasts.

\section{Patient-Specific Fibrosis Imaging}

The images with distributions of atrial fibrosis used in this study were obtained from 3 patients suffering persistent $\mathrm{AF}$ 
TABLE 1 | Electrophysiological properties of cardiac fibroblasts.

\begin{tabular}{|c|c|c|c|c|}
\hline Study & Fibroblast RMP & Currents & Cell type & Cell source \\
\hline Shibukawa et al., 2005 & $-58 \pm 3.9 \mathrm{mV}$ & $\operatorname{lns}, I_{K v}$ & Adult rat ventricle & \\
\hline Chilton et al., 2005 & - & $\mathrm{I}_{\mathrm{K} 1}, \mathrm{I}_{\mathrm{KV}}$ & Rat ventricle & Isolated \\
\hline Kiseleva et al., 1998 & $-22 \mathrm{mV}$ & - & Rat atria & Isolated \\
\hline Rook et al., 1992 & $-20 \div-30 \mathrm{mV}$ & - & Neonatal rat & Cultured \\
\hline Kamkin et al., 2003 & $-37 \pm 3 \mathrm{mV}$ & $\operatorname{lns}{ }^{*}$ & Rat atria & \\
\hline Wang et al., 2003 & - & $\mathrm{Ins}^{\dagger}, \mathrm{I}_{\mathrm{BKCa}}{ }^{\ddagger}$ & Rat ventricle & Cultured \\
\hline Miragoli et al., 2006 & $-14 \div-25 \mathrm{mV}$ & $\mathrm{I}_{\mathrm{K} 1}$ & Neonatal rat ventricle & Cultured \\
\hline Kohl et al., 2005 & - & ${ }_{\mathrm{Cl}}, I_{\mathrm{Kv}}, I_{\mathrm{K} 1}, I_{\mathrm{Na}}$ & Frog SAN & \\
\hline Kohl et al., 2005 & $-15 \mathrm{mV}$ & - & Rat atria and SAN & Isolated and Cultured \\
\hline Li et al., 2009 & - & $\mathrm{I}_{\mathrm{BKCa}}, \mathrm{I}_{\mathrm{to}}, \mathrm{I}_{\mathrm{K} 1}, \mathrm{I}_{\mathrm{Cl}}{ }^{\star *}$ & Human ventricle & Cultured \\
\hline
\end{tabular}

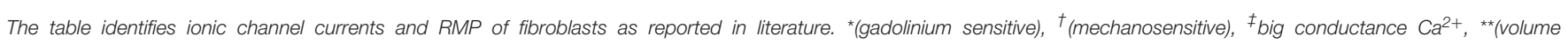
sensitive)-activated $\mathrm{K}^{+}$current has been reported in some studies of cultured cells.

and recommended for routine, first time pre-ablation imaging. These data were randomized to ensure patient privacy. A Philips 1.5T MRI scanner was used, and the images were obtained at $1.4 \times 1.4 \times 4 \mathrm{~mm}^{3}$ resolution and saved in DICOM format. Two MRI modalities were used: LGE MRI (Figures 1A-C) and early gadolinium (Gd) angiographic imaging (gated MRA) (Figure 1D). The angiography images were utilized for an improved segmentation of LGE MRI data (see details below). These were taken in the same cardiac phase (atrial diastole) with respiratory gating.

A novel image processing pipeline was developed to reconstruct and register the LGE MRI data on the fibrosis distributions with the $3 \mathrm{D}$ atria. The pipeline utilizes fibrosis segmentation tools developed by the CARMA group at the University of Utah, such as plugins to $3 \mathrm{D}$ Slicer ${ }^{\mathrm{TM}}$. This enables the reconstruction of gradual fibrosis changes as a function of image intensity.

The extraction of fibrosis data from MR images requires thresholding of the intensity of the atrial wall. Obtaining the atrial wall in MRI data is non-trivial (Varela et al., 2015) and detailed segmentation can only be performed manually due to the relatively low resolution and signal to noise ratio (SNR). This process is extremely time consuming and may provide discrepancies between segmenters. However, the LGE MR images are acquired in the same session and at the same cardiac phase as a gated MRA acquisition, used as a navigation image for planning ablation procedures. The gated MRA delineates (Figure 1D) the blood pool which has a high intensity due to the contrast agent (Gd) and allows for accurate semi-automatic segmentation of the endocardial surface. This is achieved using a region growing algorithm within ITK-Snap, which utilizes seed points that iteratively dilate. The growth is limited by image intensity gradients. The segmented endocardial surface then is dilated to provide an epicardial surface. In some cases it required manual rectification in highly convoluted areas. The dilation is usually between 3 and $5 \mathrm{~mm}$, which corresponds to the variable atrial wall thickness (Varela et al., 2015). The endocardial volume is subtracted from the dilated epicardial volume creating a 3D wall mask.

Utilising tools developed by the CARMA group and available as a 3D Slicer plugin (http://capulet.med.utah.edu/ namic/cmrslicer/), provides two different methods for fibrosis segmentation. The first, named "automatic scar segmentation," uses a K-means clustering algorithm. This produces a binary fibrosis model in the masked region, identifying the densest fibrosis areas. The second method ("threshold model") collects the MR intensity data from the masked image applying no threshold or segmentation. Both methods project the data onto a surface mesh of the endocardium. This 3D image-based atrial mesh is registered to a respective $3 \mathrm{D}$ mesh of the Visible Human Female atria using the IRTK-toolkit (https://biomedia.doc.ic.ac. $\mathrm{uk} /$ software/irtk/) using a non-linear deformation. The registered 3D mesh is projected onto the Visible Human dataset using a "nearest neighbor" algorithm. Fibrosis datasets obtained using the "threshold" tool are segmented by LGE MRI threshold, with the threshold values defined on the range of the image intensity in the masked image.

The range by LGE MRI intensity values corresponding to fibrosis were calculated individually for each patient, due to the intensity variation between scans, using the maximum intensity (MI) as the upper limit (Figure 2B). We hypothesized that the histogram intensity (Figure 2B) was a result of an overlap between two intensity distributions corresponding to healthy and fibrotic tissues (McGann et al., 2008). Thus, the right tail of the histogram was associated with fibrosis, as described in previous studies (Figure 2A), and the left tail of the histogram was associated with healthy tissue.

The range of intensity values was divided into 7 , with the lowest 2/7th being allocated to healthy tissue and each fibrosis level, 1-5 allocated as a following $1 / 7$ th of the range (moving toward higher values). Due to the densest fibrosis (level 5) covering less tissue than has previously been associated with fibrotic scars (Karim et al., 2013), the highest 1/7th of the range was expanded by $20 \%$ (moving toward lower values), 
A

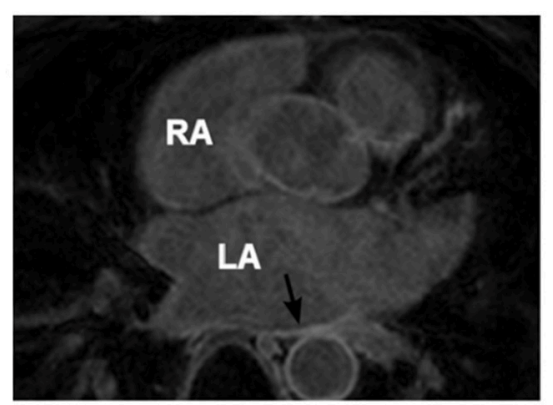

C

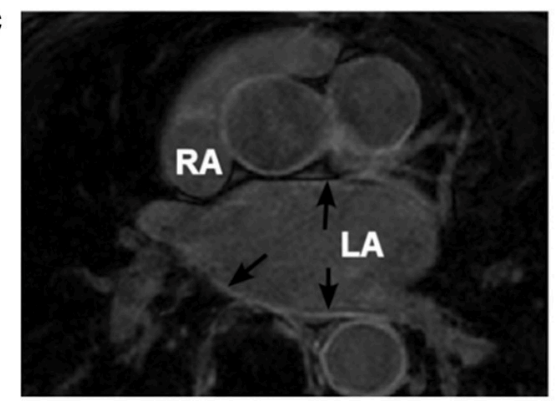

B

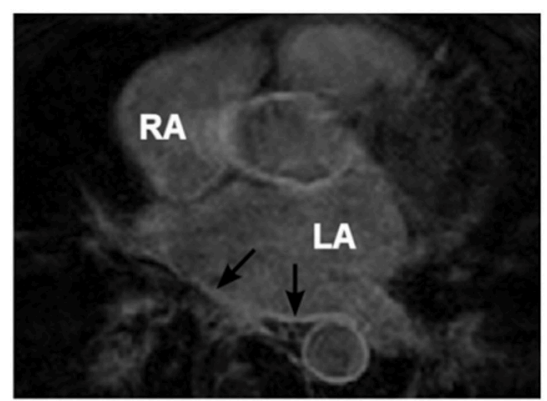

D

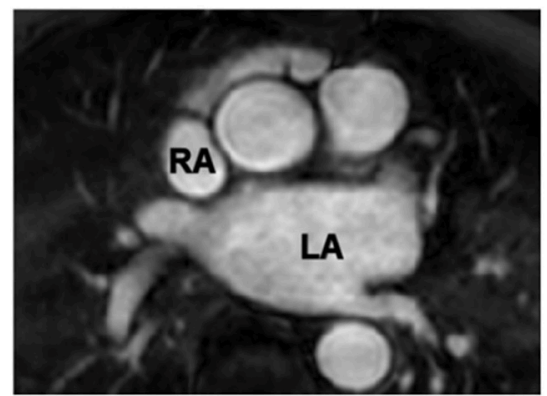

FIGURE 1 | Patient-specific MRI data. (A-C) Coronal LGE MRI slices through the atria (two atria are labelled in white) with areas of high intensity linked to fibrosis indicated by the black arrows. (D) Gd angiographic MR image with high-intensity blood volume, which is used in the segmentation of the endocardial surface. Images in panels $\mathbf{( C , D )}$ are from the same patient scan in the same coronal slice.
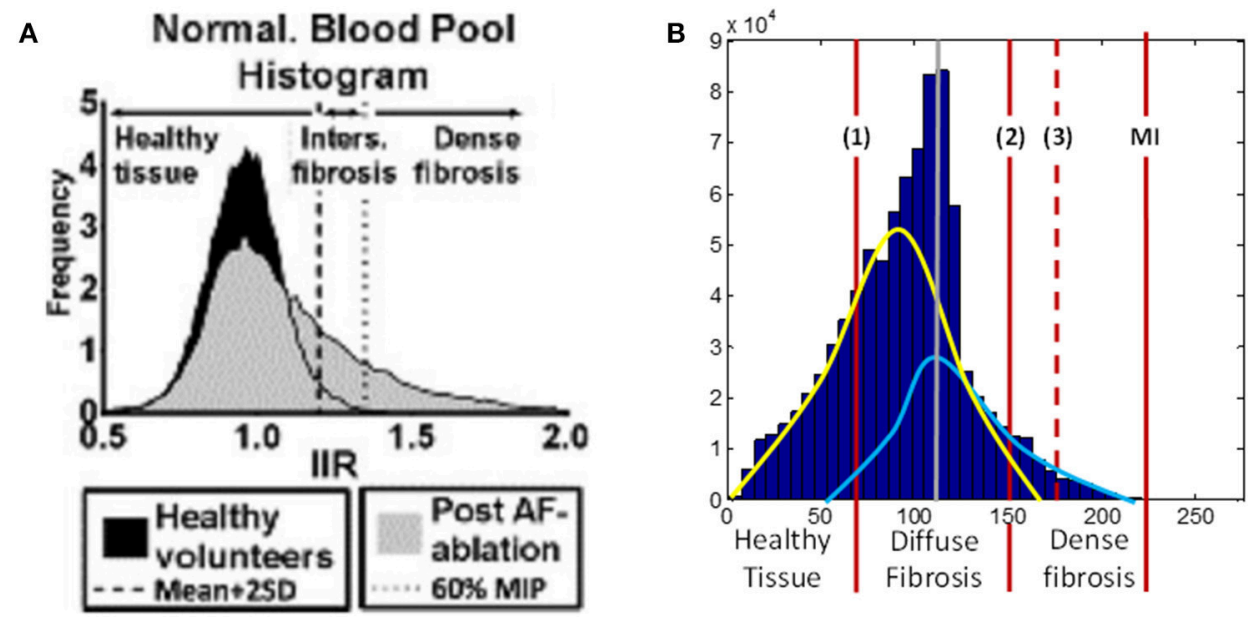

FIGURE 2 | LGE MRI intensity histograms. (A) Comparison between healthy volunteers (black shading) and fibrotic (gray shading) tissue, from Benito et al. (2015). Note that this includes the blood pool, with the intensity values normalized by the intensity of blood (hence, the peak at 1 corresponds to blood). The histogram clearly shows a tail of intensities associated with fibrosis. (B) Masked intensity histogram, i.e., only the intensity of the voxels within the masked atrial wall region. Two Gaussian curves represent approximations of the distributions of healthy (yellow) and fibrotic (light blue) tissue; the histogram peak (gray line) is the superposition maximum of these two distributions. The red lines show a range for fibrosis level segmentation. (1) is the lower limit of the diffuse fibrosis (levels 1-3) and (2) shows the lower limit for the dense fibrosis regions (levels 4, 5). The dashed red line (3) shows the lower limit of the densest (level 5) fibrosis region. The red line denoted Ml shows the maximum intensity from which the fibrosis regions were calculated. MIP, maximum intensity projection; IIR, image intensity ratio.

with all other parts of the range evenly reduced. This provided significant regions of dense fibrosis in agreement with previous reconstructions. The resultant intensity distribution between levels was as follows: healthy tissue, 0 to $0.27 \mathrm{MI}$; level 1, 0.27-0.4 MI; level 2, 0.4-0.55 MI; level 3, 0.55-0.69 MI; level 4, 0.69-0.82
MI; level 5, 0.82-1.0 MI. The segmented fibrosis regions were considered to be transmurally uniform in the $3 \mathrm{D}$ atrial model.

To investigate the role of segmentation variation, we also created two alternative segmentations for each LGE MRI dataset, one with a higher and another with a lower threshold. The higher 
threshold case in described above, and the lower threshold case was created by shifting the threshold of dense fibrosis (dashed line in Figure 2B) leftward by 10 units of LGE MRI intensity.

\section{D Atrial Simulations}

For the whole atrial simulations, the monodomain equation was solved on the 3D human atrial geometry with zero-flux boundary conditions, using the forward Euler method with spatial and temporal steps of $0.3 \mathrm{~mm}$ and $0.005 \mathrm{~ms}$, respectively. The equation is as follows:

$$
\frac{\partial V_{m}}{\partial t}=\nabla \cdot\left(D \nabla V_{m}\right)-\frac{I_{t o t}}{C_{m}}
$$

Here $\nabla$ is the gradient operator and $D$ is the diffusion coefficient, which can be a tensor when the tissue is anisotropic. The model uses the $3 \mathrm{D}$ fiber vector array to calculate the diffusion tensor to each voxel dependant on the orientation of the fiber (Clayton et al., 2011; Colman et al., 2014). This calculation also requires the longitudinal and transverse diffusion coefficients, $D_{L}$ and $D_{T}$, where $D_{L}$ was constant at $0.3 \mathrm{~mm}^{2} / \mathrm{ms}$ and $D_{T}$ was varied to change the anisotropy ratio, $R=D_{L} / D_{T}$, as described below.

The model settings were varied to simulate different conditions linked to fibrosis and AF. Four types of AF-associated remodeling were considered: (1) Ionic remodeling (Colman et al., 2013, 2014), but no structural remodeling (the standard values of $D_{L}=0.3 \mathrm{~mm}^{2} / \mathrm{ms}$ and $\left.R=10: 1\right)$, which is associated with earlystage AF. (2) Diffuse fibrosis, where instead of ionic remodeling the M-F coupling was added and inter-myocyte coupling was decreased $\left(D_{L}=0.12 \mathrm{~mm}^{2} / \mathrm{ms}, R=10: 1\right)$ throughout the atria, both changes associated with short-term AF (Rook et al., 1992; Li et al., 1999). (3) Interstitial fibrosis, where in addition to the presence of diffuse fibrosis atrial tissue anisotropy was increased $\left(D_{L}=0.12 \mathrm{~mm}^{2} / \mathrm{ms}, R=16: 1\right)$, which is associated with high level of collagenous depositions in long-term AF (Kawara et al., 2001; Krul et al., 2015). (4) Patient-specific patchy fibrosis $\left(D_{L} \leq\right.$ $0.3 \mathrm{~mm}^{2} / \mathrm{ms}, R=10: 1$ ) introduced as described below.

The segmented distinctive fibrosis levels, indexed $0-5$ depending on the LGE MRI intensity, were applied to simulate the dense-to-diffuse fibrosis gradients in the $3 \mathrm{D}$ atrial model. Index 0 represents no fibrosis, and regions with indices $1-5$ are modeled as increasingly severe fibrosis. Specifically, indices $0-$ 5 corresponded to progressively increasing M-F coupling (05 fibroblasts per myocyte) and decreasing diffusive coupling between myocytes (100-20\% of the standard $D_{L}=0.3 \mathrm{~mm}^{2} / \mathrm{ms}$ ). Thus, 3 patient-specific fibrosis distributions were generated.

The tissue effective refractory period (ERP) was calculated in a quasi-1D tissue by applying a stimulating current at one end to initiate AP propagation to the other end. This was modeled as a long $3 \mathrm{D}$ slab of $50 \times 7 \times 7$ cells with a space step of $0.3 \mathrm{~mm}$ and a diffusion coefficient of $0.3 \mathrm{~mm}^{2} \mathrm{~ms}^{-1}$. The stimulus of -2500 $\mathrm{mA}$ was applied in the first 10 layers of cells. This resulted in a $1 \mathrm{D}$ plane wave propagating along the slab. Stimuli were applied at a given BCL, followed by a last stimulus applied at a variable S2 interval. The latter was increased until an AP was observed at the non-stimulated end of the cable. The minimum S2 interval for which AP was still able to propagate through the cable was taken as ERP. These simulations also enabled the calculation of the conduction velocity $(\mathrm{CV})$ by measuring conduction times along the slab.

Re-entry was initiated in the $3 \mathrm{D}$ model through fast pacing in the RAA or LAA, allowing for the wave to propagate transversely to the crista terminalis (CT) and generate a conduction block.

\section{RESULTS}

\section{Atrial Fibroblast and M-F Coupling Models}

The fitting of ionic channel currents in the atrial fibroblast models to the respective patch-clamp data (Wu et al., 2014) can be seen in Figures 3A-D. I-V curves for $I_{K u r}, I_{t o}$ and $I_{K 1}$ (Figures 3A-C) in the model all are in good agreement with the experimental data. After fitting the ionic currents to experimental data, the RMP in the resulting model for a single atrial fibroblast was $-42.5 \mathrm{mV}$, which was in excellent agreement with the respective experimental value of $-42.8 \mathrm{mV}$ recorded from atrial fibroblasts (Wu et al., 2014). This value is more positive than the RMP of $-48 \mathrm{mV}$ in MacCannell et al. (2007) ventricular fibroblast model, although both are within the experimental data range (Table 1).

Coupling to fibroblasts significantly affected electrophysiological properties of the myocyte. Figures 4A,B, illustrates APs in the control CRN model (i) coupled to the developed atrial fibroblast model (ii), and the CRN model for a myocyte coupled to the MacCannell et al. (2007) ventricular fibroblast model (iii). In these simulations, a myocyte was coupled to 2 fibroblasts. The M-F model (ii) produced prolonged repolarisation compared to the uncoupled control model (i), while the M-F ventricular model (iii) produces a more rapid repolarization than in control. Hence, coupling to atrial (ii) and ventricular (iii) fibroblasts produces opposite effects on AP in the CRN atrial myocyte model (i). Figures 4C,D show the respective membrane potential in atrial and ventricular fibroblasts for the M-F coupling models (ii) and (iii).

The fibroblast potentials were purely electrotonic due to the coupling with the myocyte and had substantially lower amplitude than that of the myocyte. The atrial fibroblast had a slower repolarization and slightly lower amplitude than that of the coupled ventricular fibroblast. More importantly, it had a higher RMP, and hence a larger effect of the myocyte RMP.

Figure 5 compares electrophysiological characteristics of the cases (i)-(iii). The RMP (Figure 5A) was increased (became more positive) compared to control in both M-F coupling models (ii) and (iii). At a BCL of $500 \mathrm{~ms}$, in the M-F model (ii) with atrial fibroblast the RMP was $-76.5 \mathrm{mV}$ (Figure 5A) and in the $\mathrm{M}-\mathrm{F}$ model (iii) with ventricular fibroblast the RMP was $-77.5 \mathrm{mV}$, compared to $-79.8 \mathrm{mV}$ in the uncoupled CRN model (i). RMP is lowest in case (ii) throughout all BCLs, which can be particularly important at fast rates associated with AF.

The dynamic effects of coupling a CRN myocyte with the fibroblast models significantly changed the refractoriness of the myocyte. There was little difference in APD between the MF coupling model with atrial fibroblasts (ii) and the control (i) except for at a high pacing rate, e.g., at a BCL of 300 ms (Figure 5B). In comparison, the M-F coupling model with ventricular fibroblasts (iii) reduced the APD throughout the 
A

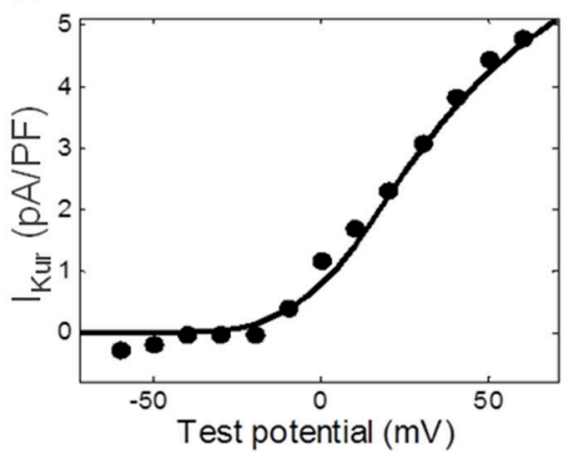

C

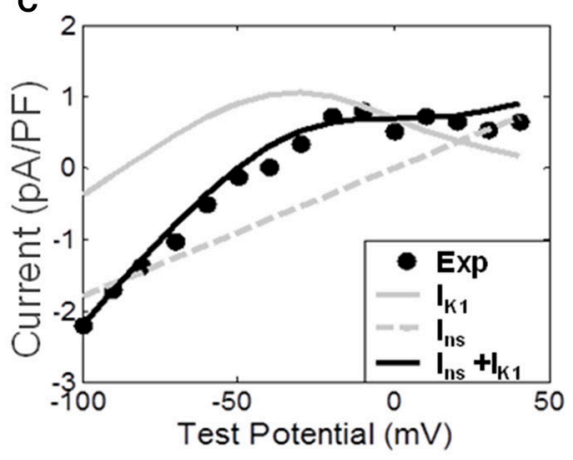

B

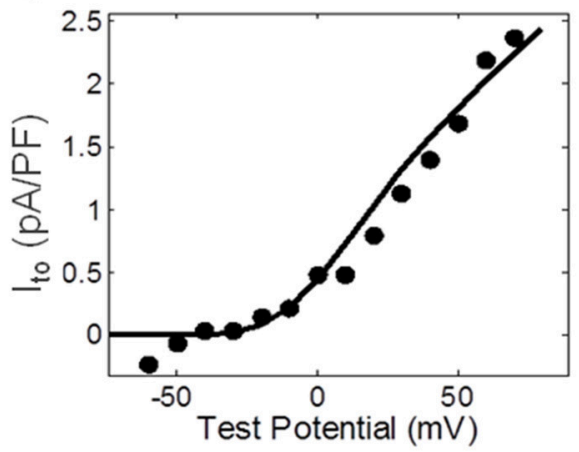

D

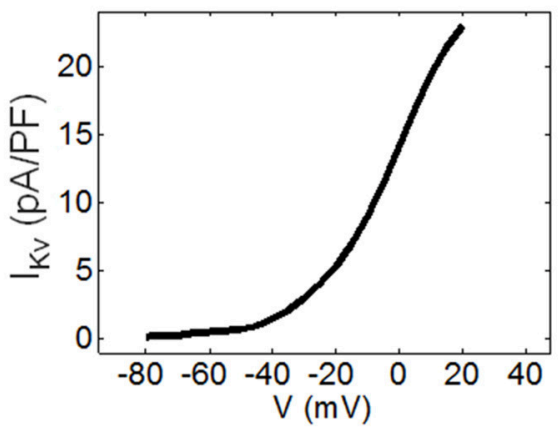

FIGURE 3 | Ionic channel currents of the atrial fibroblast model. (A-C) The simulated I-V curves for the major ionic currents (solid lines) are shown along with the respective experimental data (dots). (A) The ultra-rapid current, $I_{\text {Kur. }}$ (B) The transient outward current, $I_{t o}$. (C) The experimentally measured end-pulse current (black dots), with the simulated inward-rectifier and non-specific currents, $I_{K 1}$ and $I_{n s}$, (gray lines) and the combined current $I_{K 1}+I_{n s}$ (black line). (D) $I_{K V}$ simulated by the ventricular fibroblast model by MacCannell et al. (2007); it represent the sum of $I_{K u r}$ and $I_{\text {to. }}$ The index ' $f$ ' used in equations (1)-(4) above is omitted here for simplicity.

range of BCLs, with a maximum reduction of about $20 \mathrm{~ms}$. Effects of M-F coupling on APD became more pronounced in both models (ii) and (iii) as the number of fibroblasts coupled to a myocyte was increased from 2 to 4 and 6.

The excitation threshold for the stimulus current was also investigated (Figure 5C) for the models. In the M-F coupling model (ii) with atrial fibroblasts, the threshold was $-15.9 \mathrm{pA} / \mathrm{pF}$ at the BCL of $500 \mathrm{~ms}$. It was significantly lower when compared to both the uncoupled myocyte model (i) $(-19.2 \mathrm{~mA} / \mathrm{mS})$ and the M-F coupling model (iii) with ventricular fibroblasts $(-17.5 \mathrm{~mA} / \mathrm{mS})$. Changes of the excitation threshold in models (ii) and (iii) compared to control CRN (i) correlated with the respective changes in the RMP: higher (more positive, but lower absolute value) RMP corresponded to the lower threshold (see Figures 5A,C).

Effects of M-F coupling on ERP were larger than those on APD in both M-F coupling models (ii) and (iii). ERP in the M-F coupling model with atrial fibroblasts (ii) was larger than that in control (i) by about 5-10 ms at BCLs above $300 \mathrm{~ms}$ (Figure 5D), but dropped off rapidly at BCL was decreased below 275 ms. ERP in the M-F coupling model with ventricular fibroblasts (iii) was substantially larger than that in control (i) by about $50-100 \mathrm{~ms}$ across the range of BCLs.
Despite decreasing the excitation threshold, both M-F coupling models (ii) and (iii) reduced the CV compared to the control (i), with the ventricular M-F model producing the greater reduction. This can be explained by a substantial reduction of the sodium current, $I_{\mathrm{Na}}$, in case (ii) (Figure 5F) and by a large increase of the ERP in case (iii) (Figure 5D). The decrease of $I_{\mathrm{Na}}$ in the myocyte, particularly pronounced due to coupling with atrial fibroblasts at high rates (Figure 5F), can be explained by more positive RMP in atrial fibroblasts (Figure 5A). The latter results in a stronger electrotonic M-F current, $I_{G a p}$, (Figure 4) and partial inactivation of $I_{\mathrm{Na}}$.

Note that RMP in the novel atrial fibroblast model $(-42 \mathrm{mV})$ was more representative of atrial physiology (Table 1) than that the MacCannell et al. (2007) ventricular fibroblast model ( -47 $\mathrm{mV}$ ), as it was developed based on more comprehensive patchclamp data from atrial fibroblasts. Moreover, M-F coupling with the ventricular fibroblast model produced non-physiologically high ERP (about $350 \mathrm{~ms}$ ) and low CV $(0.4 \mathrm{~m} / \mathrm{s})$ in atrial tissue at high rates (BCL of $300 \mathrm{~ms}$ ). The high ERP in this case may be a result of slow repolarisation processes, characterized by long time constants, in the ventricular models-whereas our novel atrial fibroblast model uses formalisms, including kinetics inherited from the CRN model, specific to faster repolarisation in the atria. Hence, using the ventricular fibroblast model instead of the 

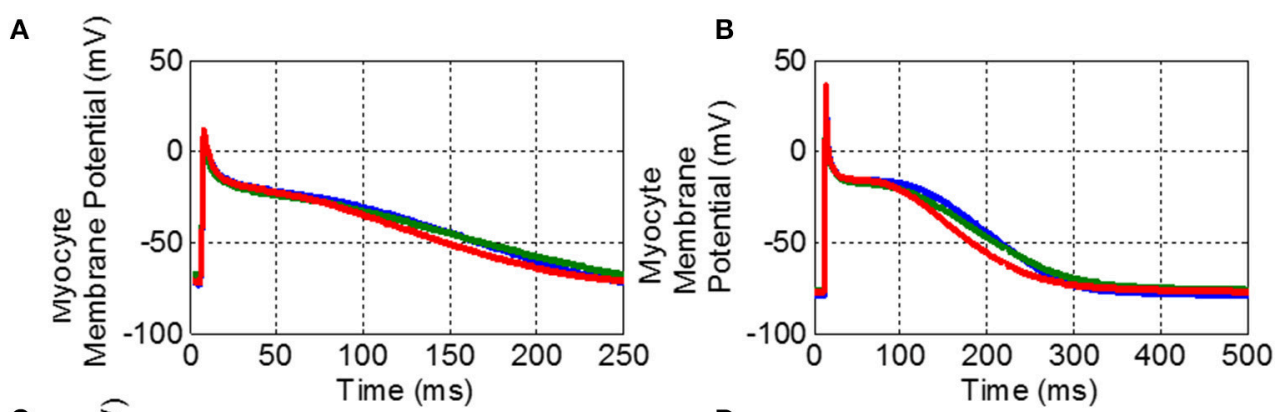

C

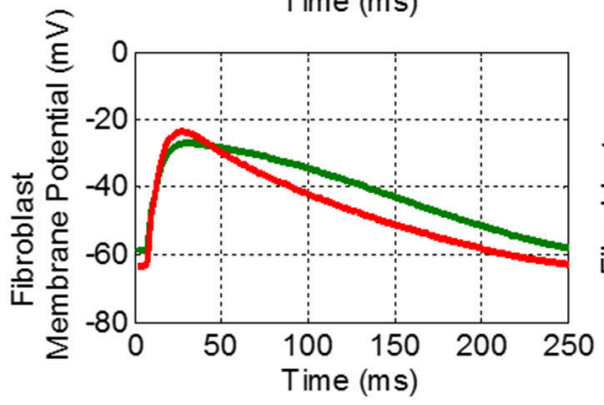

E

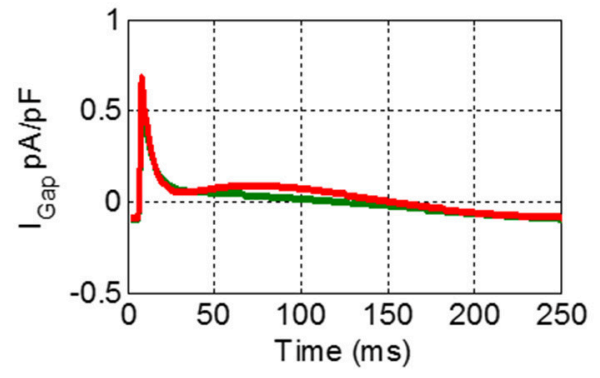

D

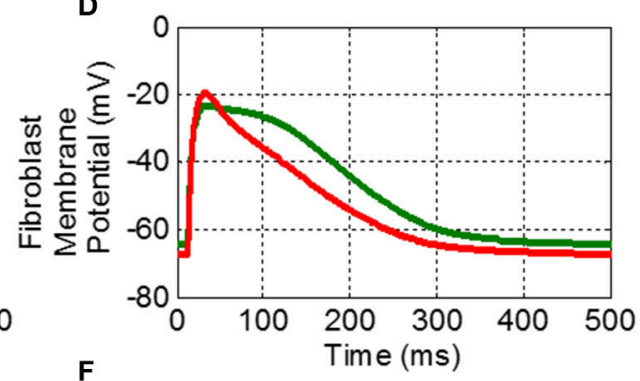

$\mathbf{F}$

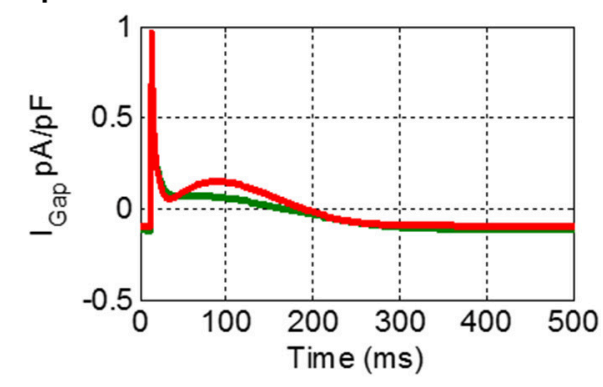

$\mathrm{CRN}-\mathrm{CRN}$ atr $-\mathrm{CRN}$ ven

FIGURE 4 | Effects of M-F coupling on AP in the atrial myocyte described by the CRN model. (A) And (B) show the AP in atrial myocyte in various conditions: uncoupled CRN (blue), coupled with the human atrial fibroblast model (green) and coupled with the ventricular fibroblast model (red). (C,D) Show the fibroblast membrane potential for the coupled models, and (E,F) show the M-F gap junctional coupling current (IGap). The left hand column (A,C, and E) shows these conditions at a BCL of $250 \mathrm{~ms}$ and the right (B,D, and $\mathbf{F})$ for a $\mathrm{BCL}$ of $500 \mathrm{~ms}$.

atrial fibroblast model in 3D human atrial models could lead to different behavior in AF, as shown in the next section.

\section{Uniform Fibrosis in 3D Atrial Models}

In $3 \mathrm{D}$ atrial simulations, fast pacing in the anterior RA resulted in a conduction block leading to wave breaks and re-entry in all simulated conditions (see Methods). The precise location and timing of the wave-block varied depending on the condition considered and pacing rate used, but was always clearly observed between the CT and the pacing site (seen in Figure 6).

In the diffuse fibrosis conditions, M-F coupling with atrial and ventricular fibroblasts resulted in greatly varying duration of re-entry: about 3 and 12 s, respectively. A much longer duration of re-entry in the latter case can be explained to the wavelength reduction associated with extremely low CV values seen with this model (Figure 5D) at high rates. The short wavelength can be seen in Figure 6 as a larger excitable gap (between a re-entering wave and the preceding one) in the case of coupling with ventricular fibroblasts (Figures 6A,C), as compared to that with atrial fibroblasts (Figures 6B,D). This also points the sensitivity of $3 \mathrm{D}$ atrial behavior to the choice of a fibroblast model, especially at high rates typical of AF.

In all diffuse fibrosis conditions, re-entry was unstable and self-terminated after several seconds (Figure 7B). In the ionic remodeling condition, known to lead to a large decrease of APD and the wavelength (Aslanidi et al., 2011; Colman et al., 2013), a single stable rotor persisted through the entire simulation (Figure 7A). Another pattern was observed in the high anisotropy condition linked with interstitial fibrosis. Reentrant waves initiated due to the wave break at the CT and a relatively short wavelength were afterwards broken down into multiple secondary wavelets (Figure 7C). The breakdown mechanism was due to the presence of a higher number of sites with slow transverse conduction (similar to the CT). The general 3D atrial activation pattern of multiple wavelets propagating quasi-chaotically and independently was typical of AF. 
A
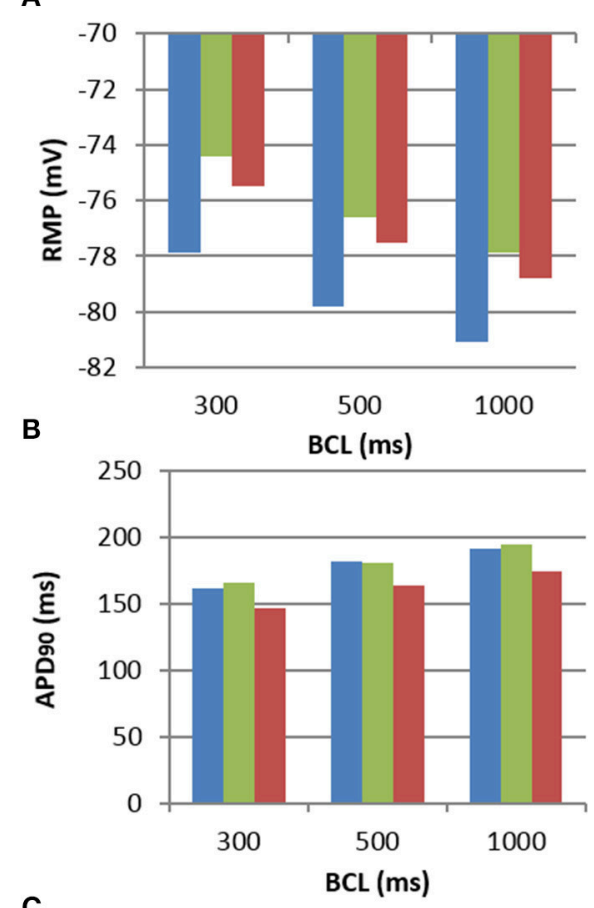

C

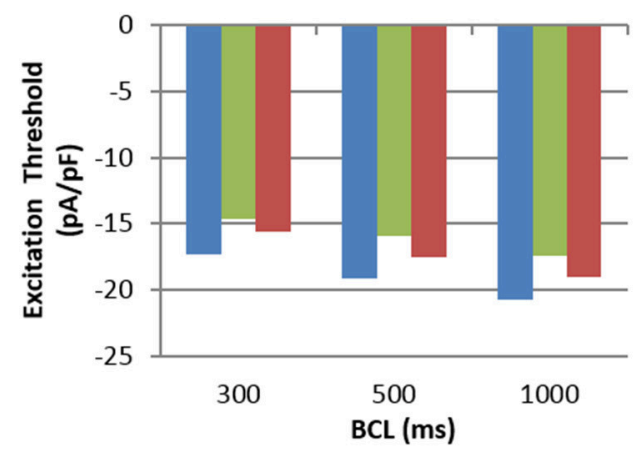

D
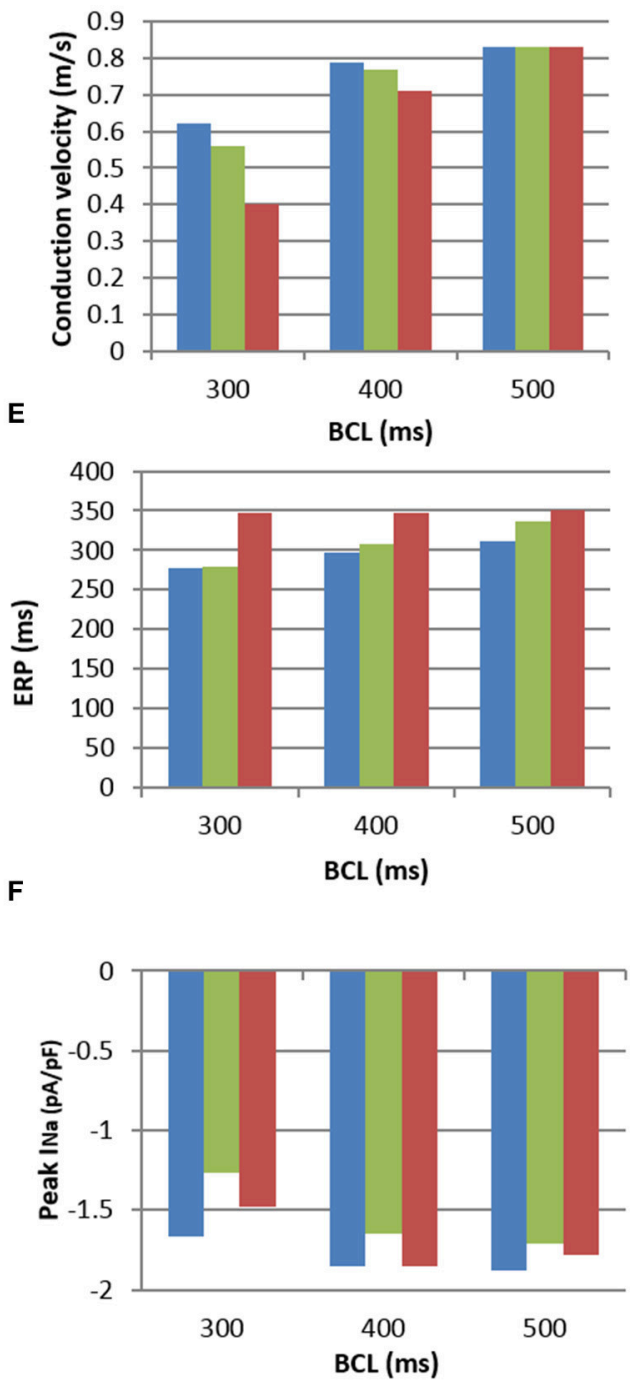

FIGURE 5 | Electrophysiological characteristics of the $\mathbf{M - F}$ coupling models. (A), RMP; (B), APD; (C), Excitation threshold in respect to the stimulating current; (D), CV; (E), ERP; (F), Peak sodium current, $\mathrm{I}_{\mathrm{Na}}$, all shown for increasing BCLs. All panels use the same colour code: uncoupled CRN myocyte model (blue), M-F coupling model with atrial fibroblasts (green) and M-F coupling model with ventricular fibroblasts (red).

However, stabilization of sustained activity in specific atrial locations was not observed in any of the uniform conditions considered. In the next section, such a stabilization of re-entrant wave is linked with patient-specific areas of heterogeneous patchy fibrosis in the $3 \mathrm{D}$ atria.

\section{Patient-Specific Fibrosis in 3D Atrial Models}

The segmentations of LGE MR images yielded different fibrosis distributions in $3 \mathrm{AF}$ patients. The reconstructed fibrosis distributions varied in the extent and severity, as seen in Figure 8. All datasets showed areas of more severe, dense fibrosis (corresponding to the highest LGE MRI intensity) in the posterior LA (Figure 8), which is a common ablation target. The relative quantized distributions of fibrosis in the thresholded datasets showed a higher distribution in the lower fibrosis levels. Significantly lower amounts of fibrosis were observed in the higher levels (4-5) despite the histogram bias for level 5 fibrosis. In all the datasets good connectivity of fibrotic regions was observed, with the fibrosis levels changing gradually (Figure 8).

In $3 \mathrm{D}$ atrial model simulations, variable duration pinning to the fibrotic region was typically seen for all patientspecific cases (Figure 9). Permanent rotor stabilization in the BZ was seen in two cases, where rotor was pinned until the end of simulation. One simulation produced rotor movement directly around an inner area of dense fibrosis (Figure 9A) and another within an adjacent border zone of less severe patchy fibrosis (Figure 9B). In both cases, the rotor core was localized within regions of broadly defined 
patchy fibrosis (levels 3-4). Spatio-temporal dynamics of the rotors is illustrated in Figures 9C,D and Supplementary Video 1.

In patient-specific 3D atrial model simulations with variation of LGE MRI intensity threshold, different behaviors were observed (Figure 10). In simulations with the higher thresholds

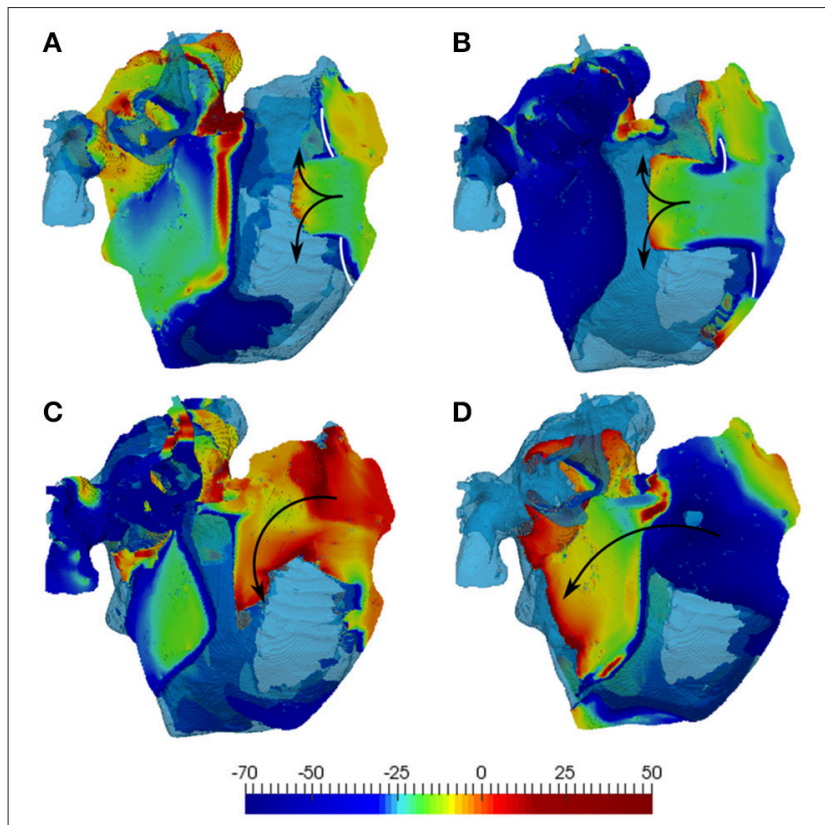

FIGURE 6 | Simulations of the 3D human atrial model with diffuse fibrosis. The latter includes normal 10:1 anisotropy, $60 \%$ reduction of diffusion coefficients and M-F coupling with 2 fibroblasts. (A) And (C) illustrate simulations with the developed atrial fibroblast model, and (B,D) show those with the MacCannell et al. (2007) ventricular fibroblast model. The top panels (A,B) shows the initial conduction blocks (white lines), and (C,D) show re-entry generation. 3D voltage maps (rainbow palette) are superimposed onto the atrial geometry (transparent blue). Wave directions in the RA are indicated by black arrows, with secondary waves travelling away into the LA. (lower fibrosis level) no stable rotor pinning was observed, with only short lived (less than $1 \mathrm{~s}$ ) meandering re-entry in the LA fibrotic regions in two patients (Figures 10A,B). Simulations of the same two patient-specific atrial models with the lower threshold (higher fibrosis level) showed severe wave breakdown, with multiple re-entrant wavelets in the LA and to a lesser extent the RA. Interactions of the wavelets in fibrotic regions prevented the generation of a single rotor (Figures 10B,D). The complex electrical activity in both patient-specific $3 \mathrm{D}$ atria was characteristic of $\mathrm{AF}$ and sustained for the entire simulation (10s).

The 3D atrial simulations performed for this section provide further evidence to the importance of using realistic fibrosis patterns to study the dynamics of AF, and elucidate the role of fibrosis in arrhythmogenesis due to the unique wave interactions for different fibrotic distributions.

\section{DISCUSSION}

In this study, cell-to-organ models of the human atria were developed and applied to explore the effects of fibrosis on AF arrhythmogenesis. Fibrosis was modeled by integrating (i) a novel electrophysiologically detailed model for a single atrial fibroblast, (ii) electrotonic myocyte-fibroblast (M-F) coupling, (iii) structural effects of fibrosis on anisotropic conductivity of atrial tissue and (iv) either uniform or patient-specific distributions of fibrosis in the 3D atrial model. Patient-specific distributions were reconstructed from clinical LGE MRI and angiography data, and a novel image processing pipeline was developed to maps the distributions of fibrosis into the $3 \mathrm{D}$ atrial model and investigate the effects of regional fibrosis patches on $\mathrm{AF}$.

The modeling revealed that: (1) electrophysiological properties of atrial fibroblasts are different from those of ventricular fibroblasts, with the largest effect of atrial M-F coupling being on the myocyte RMP and $I_{\mathrm{Na}}$, and leading to a reduction of atrial $\mathrm{CV} ; 2$ ) further to the M-F coupling,
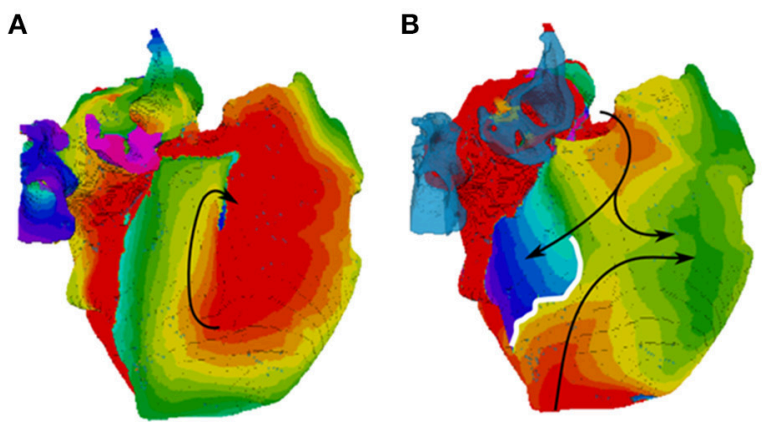

$t=0$

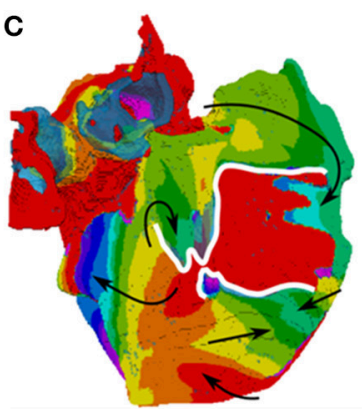

$\mathrm{t}=250 \mathrm{~ms}$

FIGURE 7 | Activation maps in the 3D atria model for typical patterns of observed re-entrant behaviours. (A) A stable rotor, seen in the conditions with ionic remodelling. (B) Unstable re-entrant waves in the conditions with diffuse fibrosis. (C) wave breakdown in the conditions with interstitial fibrosis. Wave propagation directions are shown by black arrows and wave-blocks are shown by white lines. In (B,C), the left atrium is fully activated at the initial moment of time $t=0$ and the PVs are repolarized and not activated (and hence, seen in the transparent blue color corresponding to the tissue geometry) within the range of these activation times. 


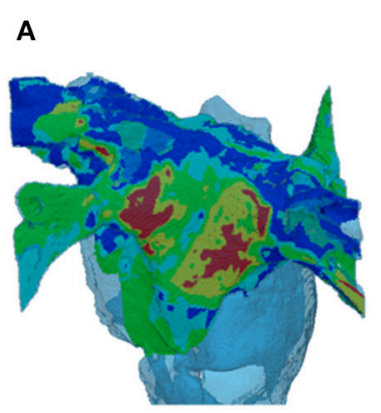

B

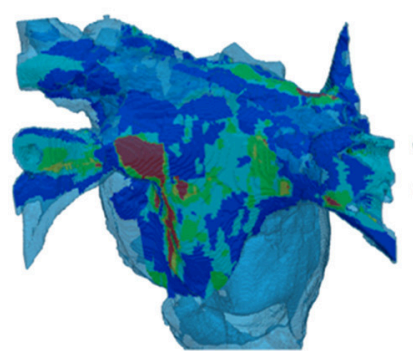

C

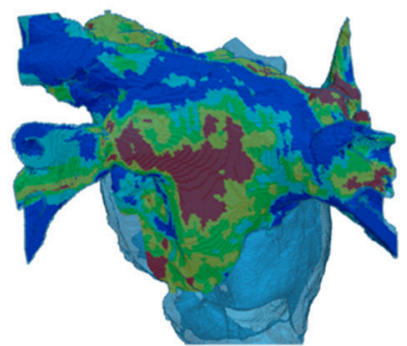

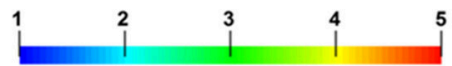

FIGURE 8 | Reconstruction of fibrosis by LGE MRI intensity threshold method from 3 AF patients. Variation in distribution can clearly be seen between patients in panels $\mathbf{( A - C ) . ~ ( A , C ) ~ I l l u s t r a t e ~ c a s e s ~ w i t h ~ l o w e r ~ t h r e s h o l d s ~ a n d ~ B ~ s h o w s ~ a ~ c a s e ~ w i t h ~ h i g h e r ~ t h r e s h o l d . ~ T h e ~ p a l e t t e ~ b e l o w ~ s h o w s ~ t h e ~ r e c o n s t r u c t e d ~ l e v e l s ~ o f ~}$ fibrosis, from diffuse (levels 1-2) to patchy (levels 3-4) and dense (level 5). 3D atrial geometry is also shown (transparent blue), similar to Figures $\mathbf{6}, \mathbf{9}$.

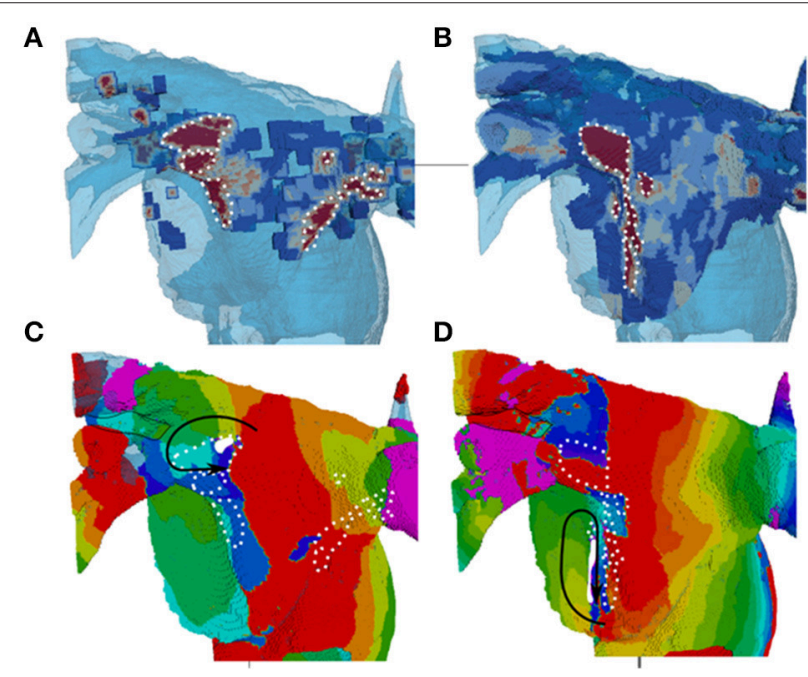

FIGURE 9 | Stabilization of rotors in patchy fibrosis regions. (A,B) Show two different patient-specific fibrosis distributions, and (C,D) Show the respective single rotor period activation maps (see color code in Figure 7). The maps illustrate the variation of rotor pinning specific to the distributions of fibrosis. Wave propagation direction is denoted by the black arrow, the core of the rotor is indicated by the solid white shape and central dense fibrosis regions are indicated by the dotted white lines. (C) Shows a rotor pinned directly to a dense fibrotic region (the dynamics of rotor movement in this case is illustrated by the Supplementary Video 1, and the respective fiber orientation in the 3D atria is shown for comparison in the Supplementary Figure 1). (D) Shows a rotor with the core adjacent to a dense fibrotic region, but rotating within a border zone.

effects of fibrosis on tissue coupling and anisotropy greatly reduce atrial $\mathrm{CV}$; 3) heterogeneous distributions of patchy fibrosis can result in rotor slowing and stabilization in fibrotic borders. The latter result provides a mechanistic explanation for the observations of recent studies (Jadidi et al., 2013; Haissaguerre et al., 2016; Zahid et al., 2016) linking patient-specific atrial fibrosis distribution and $\mathrm{AF}$ arrhythmogenesis.
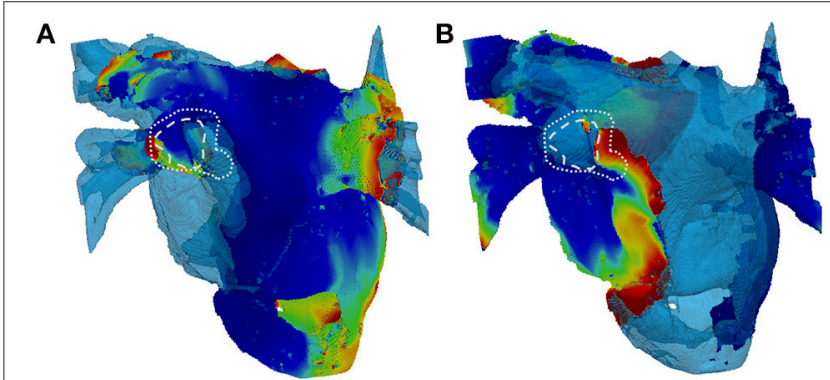

C
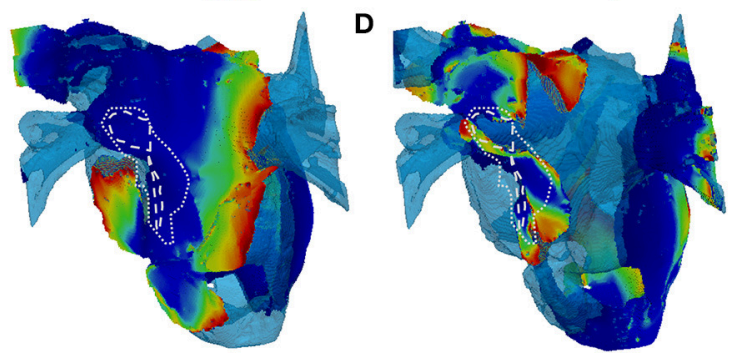

FIGURE 10 | Snapshots of re-entry in 3D atria with fibrosis distributions based on high and low thresholds of LGE MRI intensity datasets. (A,C) Show 2 patient cases with a high threshold (lower amount of fibrosis) and (B,D) Show the respective cases with a low threshold (higher amount of fibrosis). The rows delineate different patients (A-D). In the high threshold cases $(\mathbf{A}, \mathbf{C})$, only a short lived rotor (indicated) was present in the LA. In the high threshold cases (B,D), multiple re-entrant waves are present in the LA fibrotic regions. White dashed and dotted lines indicate level 5 (dense) and levels 3-4 (patchy) levels of fibrosis, respectively. 3D voltage maps are superimposed onto the atrial geometry (see color code in Figure 6).

\section{Atrial Fibroblast Model}

A novel model for atrial fibroblast electrophysiology was developed based on experimental data by Wu et al. (2014) and applied to study the effects of M-F coupling with the atrial myocyte. Atrial fibroblasts in the model had higher (more positive) RMP compared to the previous models of ventricular fibroblasts by MacCannell et al. (2007) and hence a stronger effect on the excitability of atrial myocytes. This is in agreement with 
previous studies showing that the RMP of fibroblasts can have significant effects on AP properties of the myocyte (Jacquemet and Henriquez, 2008; Maleckar et al., 2009).

During the early phases of AP, fibroblasts in the M-F model act as a current sink effectively reducing the upstroke of the myocyte, with the AP amplitude (APA) reduced by $5 \mathrm{mV}$ with the atrial fibroblast model (Figure 4). Similar behavior has been shown in other studies (Jacquemet and Henriquez, 2008; Maleckar et al., 2009), but with larger M-F coupling conductances. The APA reduction can slow down the propagation in atrial tissue due to the reduced electrotonic current transferred to neighboring myocytes. During the later phases of the AP, the fibroblasts in the M-F model act as a current source, prolonging the repolarisation phase (Figure 4) and increasing ERP (Figure 5E) in the myocyte.

The latter result is similar to the findings by Jacquemet and Henriquez (2008) and Maleckar et al. (2009) who both used a modified MacCannell et al. (2007) model for ventricular fibroblasts. However, the greatly prolonged ERP in the atrial myocyte coupled to ventricular fibroblasts (Figure 5E) is nonphysiological, and also results in non-physiologically large reductions in atrial CV (Figure 5D). Coupling to the novel atrial fibroblast model produced more physiological values of atrial ERP and CV (Figure 5). A relatively small CV decrease in this case also had a different mechanism. The excitation threshold in the myocyte coupled to atrial fibroblasts was decreased (Figure 5C) due to increased RMP in the myocyte (which was caused by inflowing $I_{\text {Gap }}$ from the atrial fibroblasts with relatively higher RMP). Counter-intuitively, the decreased threshold did not result in increased atrial CV-in fact, CV was significantly decreased (Figure 5D). This was due to the inflow of $I_{G a p}$ into the myocyte leading to a partial inactivation of $I_{\mathrm{Na}}$ (Figure 5F).

Thus, the developed model for atrial fibroblast is more relevant to atrial electrophysiology (Table 1) than the existing ventricular fibroblast models, and also provides more relevant insights into the arrhythmogenic effects of M-F coupling in the atria. The latter include CV reductions that can be responsible for regional conduction blocks and the generation of re-entrant waves. The fibroblast model provides a valuable tool for further studying $\mathrm{AF}$ in $3 \mathrm{D}$ atrial models. The model could be further validated if novel data from human atrial fibroblasts become available.

\section{D Effects of Uniform Fibrosis}

The $3 \mathrm{D}$ atria modeling enables exploring the interplay between effects of fibrosis on atrial structure and function and the resulting AF scenarios. Three uniform effects of AF-induced remodeling in the entire $3 \mathrm{D}$ atrial model were considered: ionic remodeling, diffuse fibrosis ( $\mathrm{M}-\mathrm{F}$ coupling + decreased $\mathrm{M}-\mathrm{M}$ coupling) and interstitial fibrosis (diffuse fibrosis + increased atrial tissue anisotropy). Simulations showed a range of reentrant behaviors (Figure 7).

Similar to previous studies (Colman et al., 2013), the 3D model was first validated against atrial activation data in sinus rhythm, demonstrating a good agreement with clinical measurements by Lemery et al. (2007) Further simulations of ionic remodeling effects also agreed with previous results (Aslanidi et al., 2011; Colman et al., 2013), showing a stable rotor in the 3D atrial model in the case when M-M coupling was normal (Figure 7A). This was due to the remodeling greatly decreasing atrial APD, and therefore the wavelength.

In case of diffuse fibrosis, simulations produced unstable re-entry that self-terminated after several seconds. This was due to the fact that APD and ERP in this case was relatively high (Figures 5A,E, 7B), which resulted in a long wavelength comparable to the size of the atria. Re-entry was sustained longer in the model with coupling to ventricular fibroblasts compared to atrial fibroblasts (12 vs. 3 s), due to low CV (Figure 5D), and hence the wavelength (Figure 6), in the former case. These results also point to the sensitivity of $3 \mathrm{D}$ atrial behavior to the choice of a fibroblast model, which should be relevant to atrial electrophysiology.

In case of interstitial fibrosis, AF-induced increase in tissue anisotropy (Kawara et al., 2001; Koura et al., 2002; Verheule et al., 2013; Angel et al., 2015; Krul et al., 2015) resulted in multiple conduction blocks and wave breakdown throughout the 3D atria (Figure 7C). This was the only condition where the 3D atria model produced a sustained AF-like patterns with multiple reentrant wavelets. Overall, changes of the $3 \mathrm{D}$ wave dynamics due to the increased anisotropy were more significant than the effects of M-F coupling or wavelength reduction due to ionic remodeling (Figure 7). This suggests that the structural effects of fibrosis, which develop in later stages in AF progression, may play the most important role in AF sustenance. However, stabilization of sustained activity in specific atrial locations (Haissaguerre et al., 2016), was not observed in any of the uniform cases, and can only be seen with heterogeneous distributions of fibrosis.

\section{Effects of Patchy Fibrosis in 3D Atria}

The 3D atrial simulations demonstrated unique wave interactions with different fibrotic distributions, and hence the importance of using patient-specific LGE MRI data to study AF.

The reconstructed LGE MRI patient datasets showed increased fibrosis distribution on the LA posterior wall (Figure 8). This region may be statistically prone to fibrotic infiltration and is often a site where complex fractionated electrograms are identified, which are common targets for ablation. The lower extent of fibrosis on the LA roof and floor may be due to the lower $\mathrm{Z}$ axis resolution of the MRI scan. Regardless of limitations of the imaging method, LGE MRI intensity has been strongly linked to fibrosis, and the reconstructed fibrosis distributions agree with existing knowledge from various modalities.

In $3 \mathrm{D}$ atrial simulations with patient-specific fibrosis distribution, a clear stabilization of rotors in regions of patchy fibrosis was observed in 2 out of 3 cases. One case produced a larger circus movement around a dense fibrosis region (Figure 9C) and another produced a smaller extent movement along the border of a dense region (Figure 9D). In both cases, rotors moved in slow-conducting BZ of patchy fibrosis surrounding inner regions of dense fibrosis. Note that fiber orientation can also be important in determining the rotor 
dynamics (Colman et al., 2014). However, in our simulations slow conduction in the fibrotic BZ appeared to be the main determinant of the rotor location-without patchy fibrosis, rotors meandered freely as seen in Figures 6, 7.

Further simulations also showed that patient-specific fibrosis distributions can induce wave breakdown (Figure 10) which is symptomatic of AF. This was only observed in simulation of high levels of fibrosis (obtained with a low LGE MRI intensity threshold) and resulted in permanent AF for the duration of the simulation (10 s). Smaller re-entrant wavelets were observed in these simulations moving chaotically throughout the fibrotic region. Contrarily, simulations of lower levels of fibrosis in the same patient (obtained by using a higher LGE MRI intensity threshold) produced very short duration re-entry that was neither stable nor resulted in wave breakdown. Note that the overall amount of fibrosis was not very different between the high and low thresholds. Rather, fibrosis was redistributed toward a higher proportion of severe fibrosis (levels 3-5) with an overall longer slow-conducting BZ. Hence, the rotor dynamics in $\mathrm{AF}$ may be determined primarily by the presence of such distributions.

Our results are in agreement with previous computational studies of atrial fibrosis: McDowell et al. (2015) and later Zahid et al. (2016) have shown that rotors in 3D atrial models can be localized near areas of fibrosis introduced based on LGE MRI data. Similar observations were made in cases of discrete fibrotic zones (Zahid et al., 2016), statistical distributions of fibrotic properties from dense fibrosis to healthy tissue $(\mathrm{McD}$ Dowell et al., 2015), and the respective continuous distributions in the current study. Hence, the mechanism of rotor stabilization proposed in our study can be independent on a specific way of modeling fibrosis. The accuracy of modeling fibrosis can be important for finding precise location of the rotors, and our approach of reconstructing continuous areas of patchy fibrosis may provide an advantage over models based on binary (Zahid et al., 2016) or statistical (McDowell et al., 2015) reconstructions.

\section{LIMITATIONS}

One of the major limitations of this work is a lack of experimental validation for the electrophysiological effects of fibrosis at the atrial cell and tissue level, such as its effects on APD and ERP. However, the same lack of data highlights the importance of modeling and simulation in order to better understand the problem. Further experimental measurements invivo are required for the validation of the M-F coupling effects observed in the models. Another is a lack of comprehensive clinical validation at the $3 \mathrm{D}$ atrial level, although results of our simulations are in agreement with observations of the existing studies (Jadidi et al., 2013; Haissaguerre et al., 2016; Zahid et al., 2016).

Although LGE MRI and the threshold segmentation methods also require further validation, this study aimed to investigate the effect of substantial fibrosis regions, which can be clearly seen in LGE MR images. The difference between the high and low levels of fibrosis also shows that the developed image processing and modeling pipeline is sensitive to the choice of parameters (such as LGE MRI intensity threshold) and can yield very different results. Whilst the quantification for LGE MRI intensity and amount of fibrosis from patients is currently limited, the LGE MRI intensity should indicate areas of higher fibrosis (McGann et al., 2014) in comparison to the rest of the tissue. The LGE MR images may also suffer from partial volume averaging, in which neighboring tissues influence the wall mask because of the coarse resolution of the image.

\section{CONCLUSION}

Clinically observed correlation between AF progression and fibrosis levels in the atria have led to suggestions of an empirical link between the two. The aim of this work was to understand and substantiate this link with the aid of mechanistic cell-toorgan atrial modeling. We showed that both structural and functional effects of fibrosis result in reduction of atrial $\mathrm{CV}$, and that rotors sustaining AF typically propagate in the slow conducting BZs of patchy fibrosis. These findings provide a mechanistic explanation for the improved outcomes of ablation around fibrotic areas, which can be based on the elimination of the slow-conducting substrate in the fibrotic BZ (fibrosis levels $3-4)$, and may help in future patient-specific planning of such procedures.

\section{AUTHOR CONTRIBUTIONS}

All authors have made substantial contributions to this study. $\mathrm{RM}$ and $\mathrm{OA}$ conceived and designed the study, and drafted the manuscript. RM substantially contributed to data analysis and computer simulations, and OA substantially contributed to the interpretation of the results. MC, HC, and GS contributed to data analysis, computer simulations and manuscripts editing. All authors have also approved the final version to be published while agreeing to be accountable for all aspects of the work in ensuring that questions related to the accuracy or integrity of any part of the work are appropriately investigated and resolved.

\section{FUNDING}

This work was supported by the British Heart Foundation (PG/15/8/31130).

\section{SUPPLEMENTARY MATERIAL}

The Supplementary Material for this article can be found online at: http://journal.frontiersin.org/article/10.3389/fphys. 2016.00474 


\section{REFERENCES}

Angel, N., Li, L. I., Macleod, R. S., Marrouche, N., Ranjan, R., and Dosdall, D. J. (2015). Diverse fibrosis architecture and premature stimulation facilitate initiation of reentrant activity following chronic atrial fibrillation. J. Cardiovasc. Electrophysiol. 26, 1352-1360. doi: 10.1111/jce.12773

Aslanidi, O. V., Colman, M. A., Stott, J., Dobrzynski, H., Boyett, M. R., Holden, A. V., et al. (2011). 3D virtual human atria: a computational platform for studying clinical atrial fibrillation. Prog. Biophys. Mol. Biol. 107, 156-168. doi: 10.1016/j.pbiomolbio.2011.06.011

Benito, E., Carlosena-Remirez, A., Guasch, E., Berruezo, A., Perea, R. J., Prat, S., et al. (2015). Left atrial fibrosis quantification by late gadolinium enhancement MRI: can we find the optimal normalized thresholds. Europace 17(Suppl. 3), iii89-iii91. Available online at: http://europace.oxfordjournals.org/content/17/ suppl_3/iii89.full

Calkins, H., Kuck, K. H., Cappato, R., Brugada, J., Camm, A. J., Chen, S.-A., et al. (2012). 2012 HRS/EHRA/ECAS expert consensus statement on catheter and surgical ablation of atrial fibrillation: recommendations for patient selection, procedural techniques, patient management and follow-up, definitions, endpoints, and research trial design. Heart Rhythm 9, 632-696.e21. doi: 10.1016/j.hrthm.2011.12.016

Camelliti, P., Green, C. R., LeGrice, I., and Kohl, P. (2004). Fibroblast network in rabbit sinoatrial node: structural and functional identification of homogeneous and heterogeneous cell coupling. Circ. Res. 94, 828-835. doi: 10.1161/01.RES.0000122382.19400.14

Chilton, L., Ohya, S., Freed, D., George, E., Drobic, V., Shibukawa, Y., et al. (2005). $\mathrm{K}^{+}$currents regulate the resting membrane potential, proliferation, and contractile responses in ventricular fibroblasts and myofibroblasts. Am. J. Physiol. Heart Circ. Physiol. 288, H2931-H2939. doi: 10.1152/ajpheart.01220.2004

Clayton, R. H., Bernus, O., Cherry, E. M., Dierckx, H., Fenton, F. H., Mirabella, L., et al. (2011). Models of cardiac tissue electrophysiology: progress, challenges and open questions. Prog. Biophys. Mol. Biol. 104, 22-48. doi: 10.1016/j.pbiomolbio.2010.05.008

Colman, M. A., Aslanidi, O. V., Kharche, S., Boyett, M. R., Garratt, C., Hancox, J. C., et al. (2013). Pro-arrhythmogenic effects of atrial fibrillation-induced electrical remodelling: insights from 3D virtual human atria. J. Physiol. 591(Pt 17), 4249-4272. doi: 10.1113/jphysiol.2013.254987

Colman, M. A., Varela, M., Hancox, J. C., Zhang, H., and Aslanidi, O. V. (2014). Evolution and pharmacological modulation of the arrhythmogenic wave dynamics in canine pulmonary vein model. Europace 16, 416-423. doi: 10.1093/europace/eut349

Courtemanche, M., Ramirez, R. J., and Nattel, S. (1998). Ionic mechanisms underlying human atrial action potential properties: insights from a mathematical model. Am. J. Physiol. 275, H301-H321.

Gal, P., and Marrouche, N. F. (2015). Magnetic resonance imaging of atrial fibrosis: redefining atrial fibrillation to a syndrome. Eur. Heart J. doi: 10.1093/eurheartj/ehv514. [Epub ahead of print].

Gaudesius, G., Miragoli, M., Thomas, S. P., and Rohr, S. (2003). Coupling of cardiac electrical activity over extended distances by fibroblasts of cardiac origin. Circ. Res. 93, 421-428. doi: 10.1161/01.RES.0000089258.40661.0C

Haissaguerre, M., Shah, A. J., Cochet, H., Hocini, M., Dubois, R., Efimov, I., et al. (2016). Intermittent drivers anchoring to structural heterogeneities as a major pathophysiological mechanism of human persistent atrial fibrillation. J. Physiol. 594, 2387-2398. doi: 10.1113/JP270617

Jacquemet, V., and Henriquez, C. S. (2008). Loading effect of fibroblast-myocyte coupling on resting potential, impulse propagation, and repolarization: insights from a microstructure model. Am. J. Physiol. Heart Circ. Physiol. 294, H2040H2052. doi: 10.1152/ajpheart.01298.2007

Jadidi, A. S., Cochet, H., Shah, A. J., Kim, S. J., Duncan, E., Miyazaki, S., et al. (2013). Inverse relationship between fractionated electrograms and atrial fibrosis in persistent atrial fibrillation: combined magnetic resonance imaging and high-density mapping. J. Am. Coll. Cardiol. 62, 802-812. doi: 10.1016/j.jacc.2013.03.081

Kamkin, A., Kiseleva, I., and Isenberg, G. (2003). Activation and inactivation of a non-selective cation conductance by local mechanical deformation of acutely isolated cardiac fibroblasts. Cardiovasc. Res. 57, 793-803. doi: 10.1016/S00086363(02)00775-7
Karim, R., Housden, R. J., Balasubramaniam, M., Chen, Z., Perry, D., Uddin, A., et al. (2013). Evaluation of current algorithms for segmentation of scar tissue from late gadolinium enhancement cardiovascular magnetic resonance of the left atrium: an open-access grand challenge. J. Cardiovasc. Magn. Reson. 15:105. doi: 10.1186/1532-429X-15-105

Kawara, T., Derksen, R., de Groot, J. R., Coronel, R., Tasseron, S., Linnenbank, A. C., et al. (2001). Activation delay after premature stimulation in chronically diseased human myocardium relates to the architecture of interstitial fibrosis. Circulation 104, 3069-3075. doi: 10.1161/hc5001.100833

Kiseleva, I., Kamkin, A., Pylaev, A., Kondratjev, D., Leiterer, K. P., Theres, H., et al. (1998). Electrophysiological properties of mechanosensitive atrial fibroblasts from chronic infarcted rat heart. J. Mol. Cell. Cardiol. 30, 1083-1093. doi: 10.1006/jmcc.1998.0673

Kohl, P., Camelliti, P., Burton, F. L., and Smith, G. L. (2005). Electrical coupling of fibroblasts and myocytes: relevance for cardiac propagation. J. Electrocardiol. 38, 45-50. doi: 10.1016/j.jelectrocard.2005.06.096

Kottkamp, H., Berg, J., Bender, R., Rieger, A., and Schreiber, D. (2015). Box Isolation of Fibrotic Areas (BIFA): a patient-tailored substrate modification approach for ablation of atrial fibrillation. J. Cardiovasc. Electrophysiol. 27, 22-30. doi: $10.1111 /$ jce. 12870

Koura, T., Hara, M., Takeuchi, S., Ota, K., Okada, Y., Miyoshi, S., et al. (2002). Anisotropic conduction properties in canine atria analyzed by high-resolution optical mapping: preferential direction of conduction block changes from longitudinal to transverse with increasing age. Circulation 105, 2092-2098. doi: 10.1161/01.CIR.0000015506.36371.0D

Krueger, M. W., Schmidt, V., Tobon, C., Weber, F. M., Lorenz, C., Keller, D. U. J., et al. (2011). Modeling atrial fiber orientation in patient-specific geometries: a semi-automatic rule-based approach. Lect. Note Comp. Sci. 6666, 223-232. doi: 10.1007/978-3-642-21028-0_28

Krul, S. P. J., Berger, W. R., Smit, N. W., van Amersfoorth, S. C. M., Driessen, A. H. G., van Boven, W. J., et al. (2015). Atrial fibrosis and conduction slowing in the left atrial appendage of patients undergoing thoracoscopic surgical pulmonary vein isolation for atrial fibrillation. Circ. Arrhythm. Electrophysiol. 8, 288-295. doi: 10.1161/CIRCEP.114.001752

Lemery, R., Birnie, D., Tang, A. S. L., Green, M., Gollob, M., Hendry, M., et al. (2007). Normal atrial activation and voltage during sinus rhythm in the human heart: an endocardial and epicardial mapping study in patients with a history of atrial fibrillation. J. Cardiovasc. Electrophysiol. 18, 402-408. doi: 10.1111/j.15408167.2007.00762.x

Li, D., Fareh, S., Leung, T. K., and Nattel, S. (1999). Promotion of atrial fibrillation by heart failure in dogs: atrial remodeling of a different sort. Circulation 100, 87-95. doi: 10.1161/01.CIR.100.1.87

Li, G.-R., Sun, H.-Y., Chen, J.-B., Zhou, Y., Tse, H.-F., and Lau, C.-P. (2009). Characterization of multiple ion channels in cultured human cardiac fibroblasts. PLoS ONE 4:e7307. doi: 10.1371/journal.pone.0007307

MacCannell, K. A., Bazzazi, H., Chilton, L., Shibukawa, Y., Clark, R. B., and Giles, W. R. (2007). A mathematical model of electrotonic interactions between ventricular myocytes and fibroblasts. Biophys. J. 92, 4121-4132. doi: 10.1529/biophysj.106.101410

Maleckar, M. M., Greenstein, J. L., Giles, W. R., and Trayanova, N. A. (2009). Electrotonic coupling between human atrial myocytes and fibroblasts alters myocyte excitability and repolarization. Biophys. J. 97, 2179-2190. doi: 10.1016/j.bpj.2009.07.054

Marrouche, N. F., Wilber, D., Hindricks, G., Jais, P., Akoum, N., Marchlinski, F., et al. (2014). Association of atrial tissue fibrosis identified by delayed enhancement MRI and atrial fibrillation catheter ablation: the DECAAF study. JAMA 311, 498-506. doi: 10.1001/jama.2014.3

McDowell, K. S., Zahid, S., Vadakkumpadan, F., Blauer, J., MacLeod, R. S., and Trayanova, N. A. (2015). Virtual electrophysiological study of atrial fibrillation in fibrotic remodeling. PLoS ONE. 10:e0117110. doi: 10.1371/journal.pone.0117110

McGann, C., Akoum, N., Patel, A., Kholmovski, E., Revelo, P., Damal, K., et al. (2014). Atrial fibrillation ablation outcome is predicted by left atrial remodeling on MRI. Circ. Arrhythm. Electrophysiol. 7, 23-30. doi: 10.1161/CIRCEP.113.000689

McGann, C. J., Kholmovski, E. G., Oakes, R. S., Blauer, J. J., Daccarett, M., Segerson, N., et al. (2008). New magnetic resonance imaging-based method for defining the extent of left atrial wall injury after the ablation of atrial 
fibrillation. J. Am. Coll. Cardiol. 52, 1263-1271. doi: 10.1016/j.jacc.2008. 05.062

Miragoli, M., Gaudesius, G., and Rohr, S. (2006). Electrotonic modulation of cardiac impulse conduction by myofibroblasts. Circ. Res. 98, 801-810. doi: 10.1161/01.RES.0000214537.44195.a3

Rook, M. B., van Ginneken, A. C., de Jonge, B., el Aoumari, A., Gros, D., and Jongsma, H. J. (1992). Differences in gap junction channels between cardiac myocytes, fibroblasts, and heterologous pairs. Am. J. Physiol. 263, C959-C977.

Rutherford, S. L., Trew, M. L., Sands, G. B., LeGrice, I. J., and Smaill, B. H. (2012). High-resolution 3-dimensional reconstruction of the infarct border zone: impact of structural remodeling on electrical activation. Circ. Res. 111, 301-311. doi: 10.1161/CIRCRESAHA.111.260943

Schotten, U., Verheule, S., Kirchhof, P., and Goette, A. (2011). Pathophysiological mechanisms of atrial fibrillation: a translational appraisal. Physiol. Rev. 91, 265-325. doi: 10.1152/physrev.00031.2009

Shibukawa, Y., Chilton, E. L., Maccannell, K. A., Clark, R. B., and Giles, W. R. (2005). $\mathrm{K}^{+}$currents activated by depolarization in cardiac fibroblasts. Biophys. J. 88, 3924-3935. doi: 10.1529/biophysj.104.054429

Varela, M., Kolbitsch, C., Theron, A., Morgan, R., Henningsson, M., Schaeffter, T., et al. (2015). 3D high-resolution atrial wall thickness maps using black-blood PSIR. J. Cardiovasc. Magn. Reson. 17(Suppl. 1), P239. doi: 10.1186/1532-429X17-s1-p239

Verheule, S., Tuyls, E., Gharaviri, A., Hulsmans, S., van Hunnik, A., Kuiper, M., et al. (2013). Loss of continuity in the thin epicardial layer because of endomysial fibrosis increases the complexity of atrial fibrillatory conduction. Circ. Arrhythm. Electrophysiol. 6, 202-211. doi: 10.1161/CIRCEP.112.975144

Wang, J., Chen, H., Seth, A., and McCulloch, C. A. (2003). Mechanical force regulation of myofibroblast differentiation in cardiac fibroblasts. Am. J. Physiol. Heart Circ. Physiol. 285, H1871-H1881. doi: 10.1152/ajpheart.00387.2003

Wu, C.-T., Qi, X.-Y., Huang, H., Naud, P., Dawson, K., Yeh, Y.-H., et al. (2014). Disease and region-related cardiac fibroblast potassium current variations and potential functional significance. Cardiovasc. Res. 102, 487-496. doi: $10.1093 / \mathrm{cvr} / \mathrm{cvu} 055$

Zahid, S., Cochet, H., Boyle, P. M., Schwarz, E. L., Whyte, K. N., Vigmond, E. J., et al. (2016). Patient-derived models link re-entrant driver localization in atrial fibrillation to fibrosis spatial pattern. Cardiovasc. Res. 110, 443-454. doi: $10.1093 / \mathrm{cvr} / \mathrm{cvw} 073$

Conflict of Interest Statement: The authors declare that the research was conducted in the absence of any commercial or financial relationships that could be construed as a potential conflict of interest.

Copyright () 2016 Morgan, Colman, Chubb, Seemann and Aslanidi. This is an open-access article distributed under the terms of the Creative Commons Attribution License (CC BY). The use, distribution or reproduction in other forums is permitted, provided the original author(s) or licensor are credited and that the original publication in this journal is cited, in accordance with accepted academic practice. No use, distribution or reproduction is permitted which does not comply with these terms. 